\title{
Optimization of configuration of attachment in tooth translation with transparent tooth correction by appropriate moment-to-force ratios: Biomechanical analysis
}

\author{
Yongqing Cai ${ }^{\mathrm{a},{ }^{*}}$, Bingwei $\mathrm{He}^{\mathrm{b}}$, Xiaoxiang Yang ${ }^{\mathrm{b}}$ and Jun $\mathrm{Yao}^{\mathrm{c}}$ \\ ${ }^{a}$ Department of Chemical Engineering, Fuzhou University, Fujian, China \\ ${ }^{b}$ Department of Mechanical Engineering, Fuzhou University, Fujian, China \\ ${ }^{c}$ Department of Affiliated Hospital of Stomatology, Fujian Medical University, Fujian, China
}

\begin{abstract}
Transparent tooth correction treatment is an esthetic alternative to traditional orthodontic treatment. Recently, attachments were introduced in transparent tooth correction treatments to improve the predictability of orthodontic movement. In order to optimize the attachment configuration, the force delivery system of attachments in orthodontic aligner treatment was analyzed. Based on mechanical principles, such as the synthesis theorem of force system, Varignon theorem, and the theorem of force translation, the force system of attachment was analyzed. How the attachments affected the orthodontic treatment was investigated. The attachments' force system $F_{r}, F_{t}, M_{r}$ and $M_{t}$ was determined. The optimum attachment for translation and controlled tipping movement was conceived. The attachments can be designed and placed appropriately to improve tooth movement.
\end{abstract}

Keywords: Optimization configuration, attachments, orthodontic aligner, force system

\section{Introduction}

Since its development, invisible aligner technology has become popular in worldwide orthodontic therapy as an esthetic alternative to fixed labial braces [1-4]. Initially, the aligners were used successfully to deal with mild malocclusions, such as anterior crowding and generalized spacing. However, there were controversies over whether moderate to difficult orthodontic treatment could be systematically accomplished with the orthodontic aligner system [5-8].

The orthodontic force of the orthodontic aligner is different from the traditional fixed appliance because it is derived from the rebound force of the aligner. Joffe [9] reviewed the limitations of the aligner that present the correction of moderate to severe malocclusions due to its inability to produce controlled tooth movements.

\footnotetext{
* Address for correspondence: Yongqing Cai, Department of Chemical Engineering, Fuzhou University, \#2 Xueyuan Road, Minhou County, Fuzhou City, Fujian Province, China. Tel.: +8615806033423; Fax: 0591-22866867; E-mail: caiyongqing33@126.com.
} 
Ercoli, et al. [10] compared two types of aligners. The results showed that in both aligner systems, difficulties were found in the correction of torque formation and rotations.

Align Technology, Inc recommends the use of resin attachments, interproximal reduction, thermopliers, overcorrection, or auxiliaries to aid rotational movement [11].

Rossini, et al. [12] concluded that clear aligner treatment requires the use of auxiliaries (attachments, interarch elastics, IPR, and altered aligner geometries) to improve the predictability of orthodontic movement.

Owen [13] concluded that $1 \mathrm{~mm}$ thick horizontal rectangle attachments on the buccal and lingual side help level the maxillofacial curve, extrude teeth, and control the tooth's long axis when the tooth is rotated. Boyd and Vlaskalic [14] suggested that $1 \mathrm{~mm}$ thick oval attachments perpendicular to the tooth surface for a right angle strengthen the aligner's retention.

Djeu, et al. [5] compared the treatment results of transparent teeth corrections to those done with conventional fixed appliances, using the objective grading system of the American Board of Orthodontics (ABO). Similar OGS scores between Invisalign and traditional braces for alignment and interproximal contacts were expected. It was found that Invisalign correction has had success with straightening arches by de-rotating teeth, especially when composite attachments are bonded to the premolars. In previous reports, these results were largely anecdotal; they have now been confirmed.

It was concluded that the treatment efficiencies of the orthodontic aligners and fixed appliance technology were the same. And they attributed the aligner's efficiency to attachments.

Gomez, et al. [15] concluded that the plastic aligner system's biomechanical supplementary composite attachments generate the force system required to produce bodily tooth movement; the absence of biomechanical supplementary composite attachments can result in undesired inclination of the tooth during translation movements.

Boyd [16] showed that attachments on a tooth's buccal and lingual sides can support the rotation movement. Chenin, et al. [17] reported on two cases involving the use of attachments on canine teeth and the removal of glaze from the neighboring side to assist tooth rotation. This was in accordance with reports on the effects of attachments on tooth rotation. Conversely, Kravitz, et al.'s investigation showed that attachments did not help tooth rotation [18].

It is believed that the given initial load system with certain moment-to-force ratios $(\mathrm{M} / \mathrm{F})$ can control tooth displacement [19-22]. The force system of attachment can give a clear answer on whether the introduction of attachment can help orthodontic aligner treatment. Unfortunately, there have been a limited number of investigations on how attachments work on tooth movement, and how it influences orthodontic force. Therefore, the aim of the present investigation was to use the force theorem to analyze the force system introduced by attachment in invisible aligner treatment.

\section{Materials and methods}

\subsection{Research object}

An attachment that is used to auxiliary the canine tooth's translation from the labial side to the lingual side was chosen as the research object.

There is at least one active surface involving attachments in orthodontic aligner technology, as shown in Figure 1, and the orthodontic force acting on the attachment is distributed on these surfaces. In this study, the attachment used to support the canine tooth's translation was optimized to allow effective movement of the tooth. 


\section{Non-Active Surface}

(Gap)

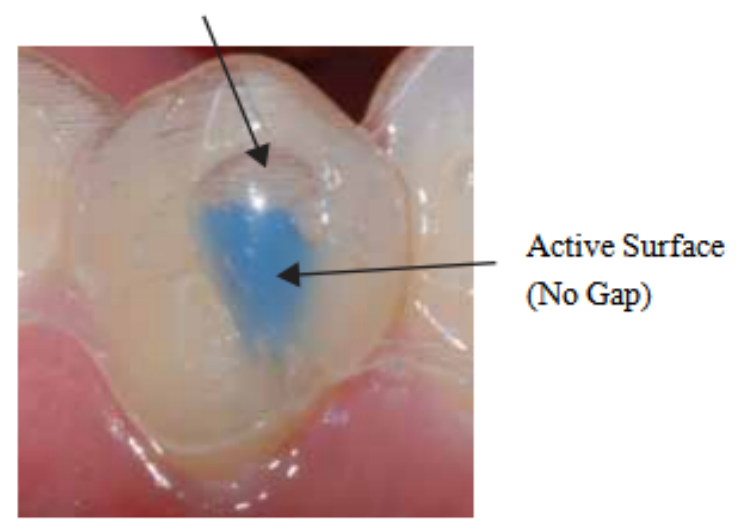

Fig. 1. Active surface of attachment.

\subsection{Optimization criterion}

There should be an optimization criterion for guiding the attachment configuration, as this can guarantee the optimization results. According to previous investigations [21, 22], the moment-to-force ratios of approximately 10 and 7 can obtain better translation and controlled tipping tooth movement. For the translation, the $\mathrm{M} / \mathrm{F}$ value was chosen as 8 in this study, and the moment and force are shown in Figure 2.

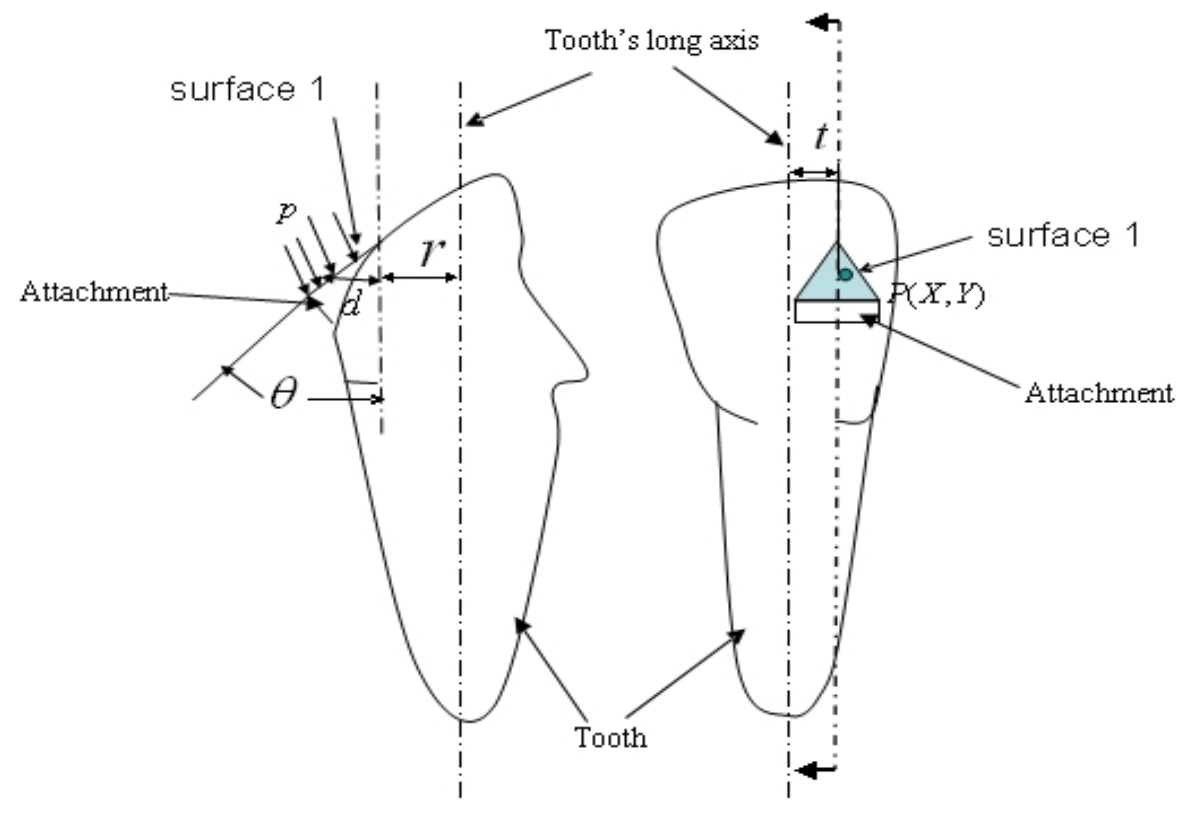

(a)

(b)

Fig. 2. Tooth and attachment. 


\subsection{Theories for analysis}

The definition of synthesis theorem of force system is that if the effect of one concentrated force action on the system is equivalent to the effect of several other forces, the concentrated force is the synthesis force of these forces.

For the theorem of force translation, the force action on the system can be translated to any other active point, but one additional moment must be added. That moment must be equal to the moment of the original force to the new active point.

According to mechanical theories, the force active on the arbitrary active surface can be simplified as a concentrated force action on the tangent plane of its active point (Figure 3). As such, the equivalent force and moment action on the tooth's long axis were obtained.

\subsection{Analysis process}

Figure 2 illustrates the attachment that was investigated in this study. Surface 1 was assumed to be the active surface in the orthodontic treatment. Thus, Surface 1 was chosen as the investigated object in the first step (Figure 3).

Friction between the aligner cavity and attachment was not addressed in this investigation, because this study mainly focused on the effect of the attachment's structure on the orthodontic force. According to the contact property, the contact pressure's direction was perpendicular to Surface 1 .

Based on the synthesis theorem of the force system, pressure $P$ (Figure 2(a)) on the surface must be equivalent to one combination force $P$ (Figure 4) acting on the surface (Eq. (1)).

$$
P=\int_{s} p d s
$$

where $P$ is the synthetic force, $p$ is the contact pressure, and $\mathrm{S}$ is the area of Surface 1.

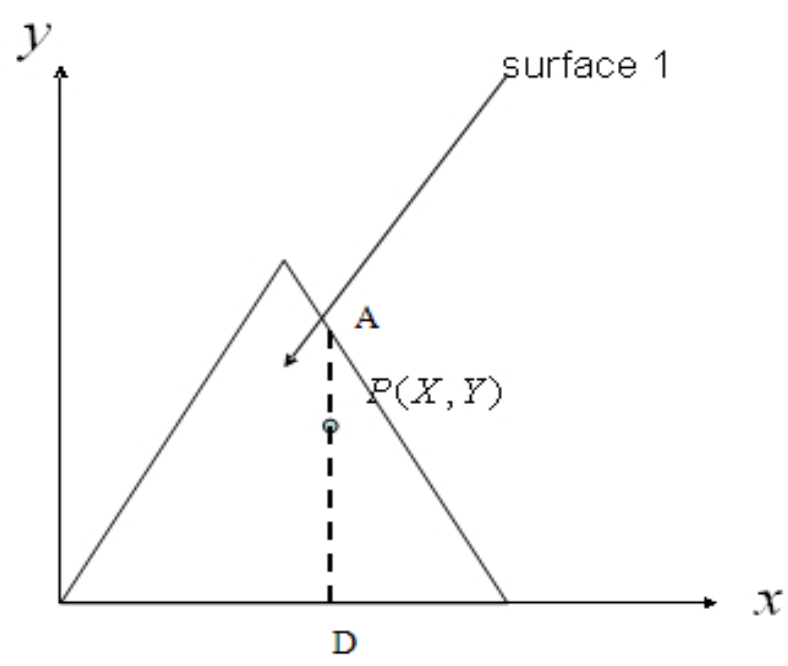

Fig. 3. Surface 1 of force active. 


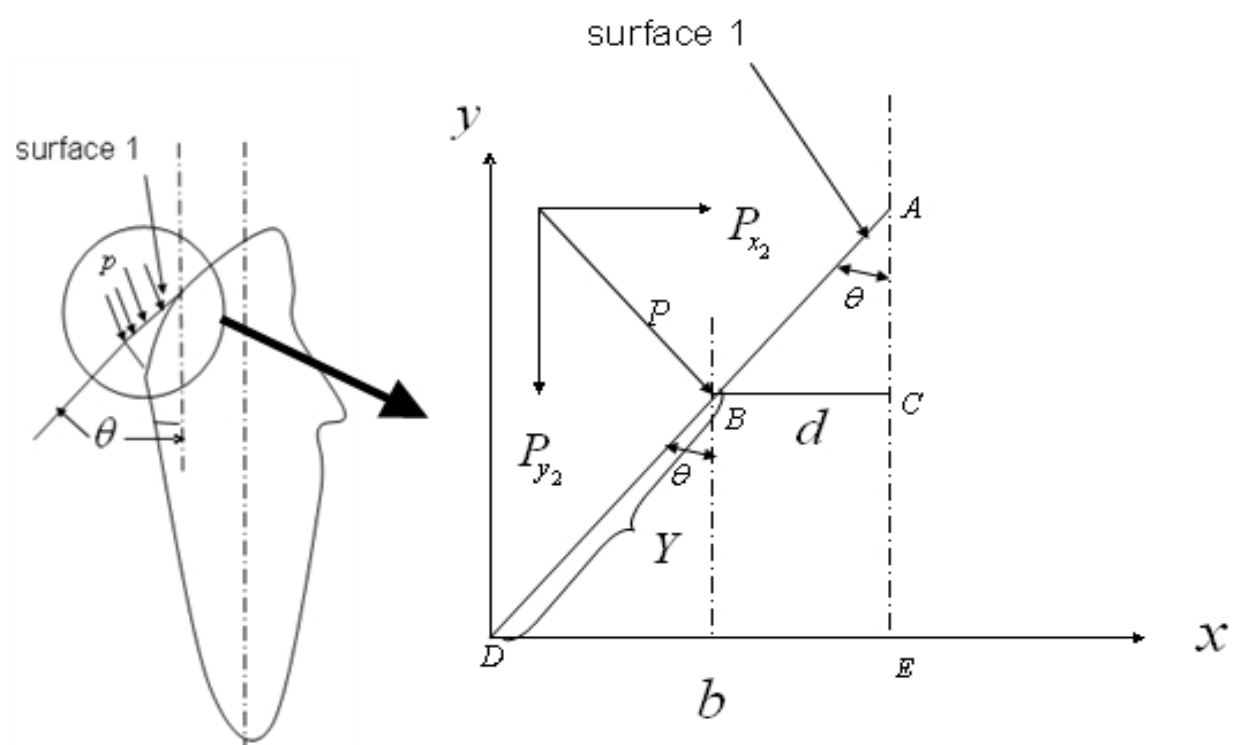

Fig. 4. Attachment investigation.

Next, the resultant moment principle was applied to obtain the coordinates of the synthetic force $P(X, Y)$.

The contact pressure's moment on $x$ axis should be equal to the moment of the synthetic force $P$ on $x$ axis, as shown in Eq. (2).

$$
\int_{s} p y \mathrm{~d} s=P Y
$$

As for the moment on $y$ axis, it was found as:

$$
\int_{s} p x \mathrm{~d} s=P X
$$

Then, the coordinates of the synthetic force $P(X, Y)$ were obtained as:

$$
X=\frac{\int_{s} p x \mathrm{~d} s}{P}, \quad Y=\frac{\int_{s} p y \mathrm{~d} s}{P}
$$

where $x$ and $y$ are the coordinates of the contact pressure, and $X$ and $Y$ are the coordinates of the synthetic force.

And in the following analysis, the contact pressure was replaced by the synthetic force. Then the attachment was taken as the investigated object (Figure 4).

The angle $\theta$ was the angle of line $A D$ in Surface 1 and the tooth's long axis. The coordinate axis $y$ in Figure 4 was parallel to the tooth's long axis.

The acting point of synthetic force $P$ in the new coordinate system was point $B$. Associated with 
Figure 3, the distance between point $B$ and $D$ was $Y$.

Then, according to the trigonometric function, coordinates of point $B$ in Figure 4's coordinate system were obtained.

$$
X_{2}=Y \sin \theta, \quad Y_{2}=Y \cos \theta
$$

Synthetic force $P$ was resolved as $P_{x_{2}}$ and $P_{y_{2}}$.

$$
P_{x_{2}}=P \cos \theta, \quad P_{y_{2}}=P \sin \theta
$$

where $X_{2}$ and $Y_{2}$ are coordinates of synthetic force $P$ in Figure 4, $P_{x_{2}}$ and $P_{y_{2}}$ are the resolution forces of force $P$ in Figure 4 .

To calculate the moment of the two resolution forces on the tooth, the distance between their active lines and the tooth's long axis was determined.

The distance $t$ between $P_{x_{2}}$ active line and the tooth's long axis was determined by the attachment's position and the active point of synthetic force $P$. The distance $R$ between $P_{y_{2}}$ active line and the tooth's long axis was determined by the crown's radium and the active point of synthetic force $P$.

Then the distance between points $B$ and $C$ needed to be determined (Figure 4). As for the similar triangles $\triangle A B C$ and $\triangle A D E, B C=d, D E=b, A D=c$, and the following was obtained:

$$
\frac{c-Y}{c}=\frac{d}{b}
$$

Then

$$
1-\frac{Y}{c}=\frac{d}{b}
$$

As for the trigonometric function, it is known that $\frac{b}{c}=\sin \theta$, so $c=\frac{b}{\sin \theta}$

Thus the following is true:

$$
1-\frac{Y \sin \theta}{b}=\frac{d}{b}
$$

Then $d=b-Y \sin \theta$

The distance between the tooth's long axis and resolution force $P_{y_{2}}$ active line is $R=d+r$, where $\mathrm{r}$ is the distance between the tooth's long axis and point $A$ (Figure 4).

Then the force and moment acting on the tooth $F_{t}, M_{t}, F_{r}, M_{r}$ is obtained by: 


$$
\begin{gathered}
F_{t}=P_{x_{2}}=P \cos \theta, \quad M_{t}=P_{x_{2}} t=P \cos \theta \bullet t \\
F_{r}=P_{y_{2}}=P \sin \theta, \quad M_{r}=P_{y_{2}} R=P \sin \theta \bullet(d+r)
\end{gathered}
$$

where $F_{t}$ is the resolution force whose direction is perpendicular to the tooth's long axis, $M_{t}$ is the additional moment of $F_{t}$ on the tooth, $F_{r}$ is the resolution force parallel to the tooth's long axis, $M_{r}$ is $F_{r}$ 's additional moment on the tooth.

\section{Results}

\subsection{Theory derived results}

There were four moment-to-force ratios:

$$
\begin{gathered}
\frac{M_{t}}{F_{t}}=\frac{P \cos \theta \bullet t}{P \cos \theta}=t \quad \frac{M_{t}}{F_{r}}=\frac{P \cos \theta \bullet t}{P \sin \theta}=\frac{t}{\tan \theta} ; \\
\frac{M_{r}}{F_{r}}=\frac{P \sin \theta \bullet(d+r)}{P \sin \theta}=d+r \quad \frac{M_{r}}{F_{t}}=\frac{P \sin \theta \bullet(d+r)}{P \cos \theta}=\tan \theta \bullet(d+r)
\end{gathered}
$$

For $d=b-Y \sin \theta$; the following was obtained:

$$
\frac{M_{r}}{F_{t}}=b-Y \sin \theta+r \quad \frac{M_{r}}{F_{t}}=\tan \theta(b-Y \sin \theta+r)
$$

The forces and moments $F_{t}, F_{r}, M_{t}$ and $M_{r}$ are shown in Figures 5 and 6.

\subsection{Optimization results}

Moment-to-force ratios are particularly useful because they have been linked to types of tooth movements. In Figures 5 and 6, it was discovered which force or moment should be retained or eliminated for different types of tooth movements. The moment-to-force ratios of approximately 10 and 7 were linked to translation and controlled tipping, respectively [21, 22].

In this study, the translation movement was taken as an example. In the translation case, the $M_{t}$ and $F_{r}$ should be eliminated, or kept as small as possible (Figure 7), and the ratio of $\frac{M_{r}}{F_{t}}$ should range from 7 to 10. The load applied to the tooth and the movements of the tooth are shown in Figure 7.

As for moment $M_{t}$, the attachment can be placed where the active line of $P_{x_{2}}$ is intersected with the tooth's long axis, which means $\mathrm{t}$ is zero and $M_{t}$ is zero. As for the force $F_{r}$, two different active 


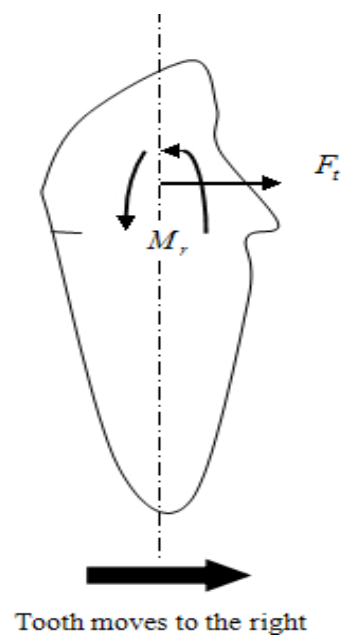

Fig. 5. Load applied to tooth and tooth movement.

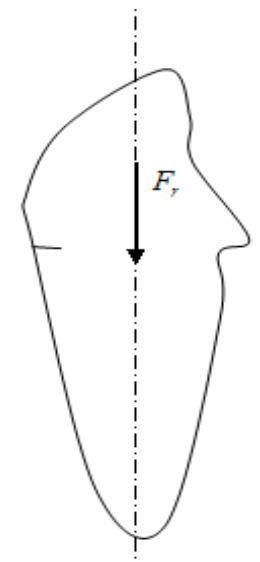

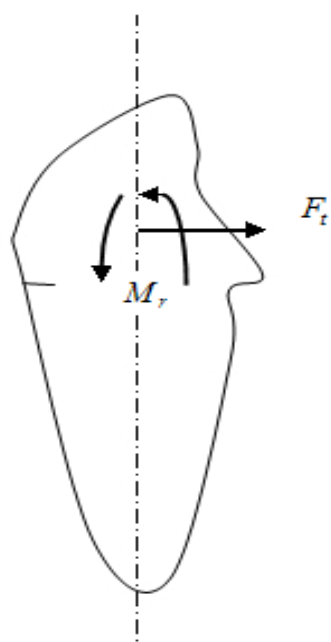

Fig. 6. Moment $M_{r}$ and force $F_{t}$.

Fig. 7. Moment $M_{t}$ and force $F_{r}$.

surfaces can be designed to make $F_{r}$ 's direction opposite. Consequently, the moment $M_{r}$ can be retained and the force $F_{r}$ can be eliminated.

Usually, the thickness of the attachment is approximately $1 \mathrm{~mm}$. If its value is too large, the aligner would be difficult to apply. Therefore, the value of $b$ is assumed to be approximately 1 .

The direction of the active surface was determined by $\frac{M_{r}}{F_{t}}=\tan \theta(b-Y \sin \theta+r)$, $\left(0^{0}<\theta<90^{\circ}, b \leq 1 \mathrm{~mm}\right)$. Because of the trigonometric function (Figure 4), $Y<\frac{b}{\sin \theta}$, and $Y \sin \theta<b \leq 1 \mathrm{~mm}$. Then it was assumed that $b-Y \sin \theta+r \approx r$. Usually the radius of the tooth's crown $r$ is about 2-4 mm, so $t \approx 4$ was used in this investigation. Then the ratio of $\frac{M_{r}}{F_{t}} \approx \tan \theta \bullet 4$ was obtained. Here it was assumed that the ratio value was 8 . Then it was known that 
$\tan \theta \bullet 4 \approx 8$, and the appropriate value of the angle was found as $\theta \approx 63.43^{0}$.

After that, the position of the attachment, its thickness, and the active surface's direction of the attachment were all determined. The attachment's design was complete.

\section{Discussion}

The biomechanical characteristics are crucial for tooth movement in orthodontic treatment systems. The limitations of the biomechanical characteristic are the essence of the limitations of the efficacy and tooth movement in orthodontic treatment.

In transparent tooth correction movement, an intentional, predetermined "mismatch" between the aligner and the tooth is programmed in each treatment stage, using a cast or virtual model set-up (alignment). The aligner, which corresponds to the new desired tooth position, is fitted on the dental arch, producing in each mismatch a force system that is directly transmitted to the tooth, generating the mechanical-biological chain of events that results in a new position of the tooth [15]. The interaction between the aligner and the tooth is complicated because of the complex and irregular shape of the aligner. The aligner attaches to tooth crown's surface to apply orthodontic forces, which may act on any position of the crown's surface. It is difficult to determine the location where the orthodontic forces will act on, and what kinds of forces and moments will be introduced. Simon, et al. [23] concluded that moment transfer from the removable appliances to the tooth is possible only to a limited degree. Moreover, Hahn, et al. [24] found the forces and couples delivered by aligners are determined by the shape of the crown and the type and amount of displacement of the particular tooth and, therefore, the contrasts between the particular tooth and the inner surface of the appliance. That may cause an inability to control the types of tooth movements. And Joffe [9] believed that led to limitations of orthodontic aligners.

The introduction of attachment can solve this type of problem. Firstly, the location the orthodontic forces act on can be determined. This is mainly decided by the position of the attachment. In this aspect, the function of attachment in orthodontic aligner treatment is similar to that of a bracket in traditional fixed orthodontic treatment. And therefore the efficiency of aligner treatment with attachments should be similar to that in traditional treatment. This opinion was supported by the research of Djeu, et al. [5].

However, Kravitz, et al. [18] thought the attachment could not help tooth movement. This could happen because of the second problem that was not made clear. What kind of orthodontic force and moment were introduced? In Kravitz, et al.'s investigation, the orthodontic force and moment delivered by the attachment may not meet his designed tooth movement.

This investigation was conducted to solve this problem. According to the derived result and the experience value of the moment-to-force ratio for tooth movement, the attachment in this study was optimized. The introduction of attachment can help tooth movement; however, its position and configuration are the key determinants.

Attachments used for other teeth and other kinds of tooth movements can also be optimized by the same method in this study. For example, the movement of rotation along the tooth's long axis and the moments $M_{t}$ and $F_{r}$ were the main parameters used to optimize the auxiliary attachment, and the analysis process would be the same as above. 


\section{Conclusion}

Analysis of the attachment force system showed that the attachment could be optimized to help the tooth move effectively. And the most important factor affecting the attachment's efficiency was found to be the active surface's direction, which can be optimized by the moment-to-force ratio needed by the movement. With the use of attachments and optimized parameters of such, it was found that orthodontic aligner treatments could offer results similar to those of traditional tooth-straightening systems.

\section{Acknowledgment}

This work was supported by the Project of Fujian education department (WKJ-FJ-02) and Project of Fujian provincial health department (JA11010), China.

\section{References}

[1] R.L. Boyd, Esthetic orthodontic treatment using the invisalign appliance for moderate to complex malocclusions, Journal of Dental Education 72 (2008), 948-967.

[2] N.D. Kravitz, B. Kusnoto, E. BeGole, A. Obrez and B. Agran, How well does Invisalign work? A prospective clinical study evaluating the efficacy of tooth movement with Invisalign, American Journal of Orthodontics and Dentofacial Orthopedics 135 (2009), 27-35.

[3] E. Kuo and R.J. Miller, Automated custom-manufacturing technology in orthodontics, American Journal of Orthodontics and Dentofacial Orthopedics 123 (2003), 578-581.

[4] B. Meier, K.B. Wiemer and R.R. Miethke, Invisalign-patient profiling, analysis of a prospective survey, Journal of Orofacial Orthopedics-fortschritte Der Kieferorthopadie 64 (2003), 352-358.

[5] G. Djeu, C. Shelton and A. Maganzini, Outcome assessment of Invisalign and traditional orthodontic treatment compared with the American board of orthodontics objective grading system, American Journal of Orthodontics and Dentofacial Orthopedics 128 (2005), 292-298.

[6] A.M. Bollen, G.J. Huang, G. King, P. Hujoel and T. Ma, Activation time and material stiffness of sequential removable orthodontic appliances, Part I: ability to complete treatment, American Journal of Orthodontics 124 (2003), 496-501.

[7] K. Clements, A.M. Bollen, G.J. Huang, G. King, P. Hujoel and T. Ma, Activation time and material stiffness of sequential removable orthodontic appliances, Part II: dental improvements, American Journal of Orthodontics 124 (2003), $502-508$.

[8] M.G. Taylor, S.P. McGorray, S. Durrett, S. Pavlow, N. Downey, M. Lenk, D. Oxford, C. Dolce and T.T. Wheeler, Effect of Invisalign aligners on periodontal tissues, Journal of Dental Research 82 (2003), 1483.

[9] L. Joffe, Current Products and Practice, Invisalign: Early experiences, Journal of Orthodontics 30 (2003), 248-252.

[10] F. Ercoli, M. Tepedino, V. Parziale and C. Luzi, A comparative study of two different clear aligner systems, Progress in Orthodontics 15 (2014), 31-36.

[11] O.C. Tuncay, The invisalign system, Quintessence Publishing Co. Ltd., London, 2006, pp. 121-132.

[12] G. Rossini, S. Parrini, T. Castroflorio, A. Deregibus and C.L. Debernardi, Efficacy of clear aligners in controlling orthodontic tooth movement: A Systematic review, Angle Orthodontist Nov. (2014)

[13] A.H 3rd. Owen, Accelerated invisalign treatment, Journal of Clinical Orthodontics 35 (2001), 381-385.

[14] R. Boyd and V. Vlaskalic, Orthodontic treatment of a mildly crowded malocclusion using the invisalign system, Australian Orthodontic Journal 17 (2001), 41-46.

[15] J.P. Gomez, F.M. Peña, V. Martínez, D.C. Giraldo and C.I. Cardona, Initial force system during bodily tooth movement with plastic aligners and composite attachments: A three-dimensional finite element analysis, Angle Orthodontist $\mathbf{8 5}$ (2015), 454-460.

[16] R.L. Boyd, Surgical-orthodontic treatment of two skeletal Class III patients with Invisalign and fixed appliances, Journal of Clinical Orthodontics 39 (2005), 245-258.

[17] D.A. Chenin, A.H. Trosien, P.F. Fong, R.A. Miller and R.S. Lee, Orthodontic treatment with a series of removable appliances, Journal of the American Dental Association 134 (2003), 1232-1239.

[18] N.D. Kravitz, B. Kusnoto, B. Agran and G. Viana, Influence of attachments and interproximal reduction on the accuracy 
of canine rotation with Invisalign: A prospective clinical study, Angle Orthodontist 78 (2008), 682-687.

[19] C.J. Burstone and R.J. Pryputniewicz, Holographic determination of centers of rotation produced by orthodontic forces, American Journal of Orthodontics 77 (1980), 396-409.

[20] P. Gjessing, Controlled retraction of maxillary incisors, American Journal of Orthodontics and Dentofacial Orthopedics 101 (1992), 120-131.

[21] J.C. Bennett and R.P. McLaughlin, Orthodontic treatment mechanics and the preadjusted appliance, Mosby Elsevier Health Science, London, 1993.

[22] R. Nanda and A.J. Kuhlberg, Principles of biomechanics, in: Biomechanics in Clinical Orthodontics, R. Nanda, ed., Saunders, St Louis, 1997, pp. 7-8.

[23] M. Simon, L. Keilig, J. Schwarze, B.A. Jung and C. Bourauel, Forces and moments generated by removable thermoplastic aligners: Incisor torque, premolar derotation, and molar distalization, American Journal of Orthodontics and Dentofacial Orthopedics 145 (2014), 728-736.

[24] W. Hahn, A. Zapf, H. Dathe, J. Fialka-Fricke, S. Fricke-Zech, R. Gruber, D. Kubein-Meesenburg and R. Sadat-Khonsari, Torquing an upper central incisor with aligners--acting forces and biomechanical principles, European Journal of Orthodontics 32 (2010), 607-613. 